\title{
Context-aware multicast routing scheme for Disruption Tolerant Networks
}

\section{Mooi-Choo Chuah* and Peng Yang}

\author{
Department of Computer Science and Engineering, \\ Lehigh University, PA 18015, USA \\ E-mail: chuah@cse.lehigh.edu \\ E-mail: pey204@lehigh.edu \\ ${ }^{*}$ Corresponding author
}

\begin{abstract}
Disruption Tolerant Networks (DTNs) are designed to enable communications in networks that experience frequent network partitions and large end-to-end delays. In this paper, we propose a Context-aware Adaptive Multicast Routing (CAMR) scheme for DTNs. Simulation results show that our CAMR scheme can achieve higher message delivery ratio with similar delay performance than two existing DTN multicast schemes, namely DTBR and OS-Multicast. We evaluate the delivery performance of CAMR in different scenarios e.g., different number of groups, different maximum node speeds. Our results indicate that CAMR scales well and provides excellent delivery performance in many different scenarios.
\end{abstract}

Keywords: disruption tolerant networking; adaptive routing; node-density; multicast.

Reference to this paper should be made as follows: Chuah, M-C. and Yang, P. (2009) 'Context-aware multicast routing scheme for Disruption Tolerant Networks', Int. J. Ad Hoc and Ubiquitous Computing, Vol. 4, No. 5, pp.269-281.

\begin{abstract}
Biographical notes: Mooi-Choo Chuah received her $\mathrm{PhD}$ in Computer Science from University of California, San Diego. She is currently an Associate Professor in the Department of Computer Science and Engineering at Lehigh University. Her research interests are in heterogeneous network architecture design, resource management, network security, disruption tolerant network design, and adhoc/sensor networks. She runs the WiNS laboratory at Lehigh University.

$\mathrm{P}$. Yang is a $\mathrm{PhD}$ candidate in the Department of Computer Science and Engineering at Lehigh University. He received his MEng in Computer Engineering from Nanyang Technological University, Singapore and a BEng in Computer Engineering from the Huazhong University of Science and Technology, China. His current research focuses on architectural and protocol design for delay/disruption tolerant networks.
\end{abstract}

\section{Introduction}

There are emerging network scenarios where an instantaneous end-to-end path between a source and destination may not exist because links between nodes may be opportunistic. New architecture and communication protocols need to be designed to allow nodes in such challenging network environments to communicate with one another. There is ongoing research (Zhao et al., 2005; DARPA, 2005; Fall, 2004; Chuah et al., 2005) on Disruption Tolerant Networks (DTNs) that addresses such challenges. DTNs have a broad range of potential applications e.g., military battlefields (Malladi and Agrawal, 2002), vehicular communications (Wu et al., 2004; . Burgess et al., 2006), deep-space communications (Burleigh et al., 2003), habitat monitoring (Cerpa et al., 2001), and internet access in rural areas (Jain et al., 2004).

Some DTN applications need multicast services. For example, in military battlefields, a platoon leader may want to distribute local map to his platoon members. For layman application scenarios, DTN multicast services allow information about victims and potential hazards be disseminated among rescue workers in disaster recovery events. However, traditional multicast methods proposed for the Internet (e.g., MOSPF (Moy, 1994) and DVMRP (Waitzman et al., 1988) or mobile ad hoc networks (e.g., AMRoute (Xie et al., 2002) and ODMRP (Bae et al., 2000) are not suitable for DTNs for several reasons. First, many of these schemes assume a multicast tree can be established but it is difficult to maintain such a tree during the lifetime of a multicast session in DTN environment. Second, message delivery may fail due to the frequent network partitions and sparse connectivity. In fact, the traditional approaches may fail to deliver a message when the communication link is highly unavailable (e.g., $~ 80 \%$ ).

To address these issues, several DTN multicast approaches have been proposed e.g. (Zhao et al., 2005), (Ye et al., 2006). In Zhao et al. (2005), the authors 
investigate several multicast routing algorithms e.g., Unicast Multicast (U-Multicast), Static Tree-Based Routing (STBR), Dynamic Tree-Based Routing (DTBR), a unicast-based routing, and group-based routing. In (Ye et al., 2006), the authors propose an on-demand situation-aware multicast (OS-multicast) approach which is a dynamic tree-based method that integrates DTN multicasting with the situation discovery mechanism provided by the underlying network layer. Their simulation results show that OS-multicast can achieve smaller delays and better message delivery ratios than other schemes e.g., DTBR at low load. OS-multicast also achieves higher efficiency when the probability of link unavailability is high and the duration of link downtime is large. However, these two approaches rely on the opportunistic connectivity among nodes for delivery without making use of additional information like node location and node velocities. We are interested in understanding the performance improvement one can get from a multicast routing scheme that makes use of such information. We also want to design a scheme that is adaptive to the network environments that are dynamically changing e.g., densely connected nodes in the battlefield may become very sparsely connected when they face obstacles while they move around. Thus, in this paper, we design a node-density based adaptive multicast routing scheme for DTNs that uses information like node location and velocities. In our approach, we assume that nodes can be turned into message ferries when circumstances, e.g., partitions of nodes, mandate such role changes. Other researchers have made a similar assumption of such dynamic role change of nodes (e.g., Dai and Wu, 2007). Then, via simulations, we compare our CAMR scheme with U-Multicast, DTBR, and OS-Multicast schemes. Our results indicate that CAMR achieves the highest delivery ratio with good data efficiency. The price to pay is some decrease in data efficiency where data efficiency is defined as the total unique multicast packets received over the total number of transmitted data packets.

The rest of the paper is organised as follows. In Section 2, we summarise related works on designing efficient DTN routing schemes. In Section 3 we present descriptions of the network model and multicast model of DTNs. In Section 4, we describe some existing multicast approaches for DTNs which include DTBR and OS-Multicast schemes. We also describe our CAMR scheme. Simulation set up and performance evaluations of different multicast schemes are presented in Section 5. Section 6 summarises our contributions.

\section{Background and related work}

There are many examples of occasionally-connected networking environments e.g., deep-space interplanetary communications (Burleigh et al., 2003). In interplanetary communications, the communication links may be available only for certain periods of time. Communications in the battlefield are another category of examples. Here, the network may be partitioned due to node mobility or geographical barriers. Wireless sensor networks deployed in harsh environments are another set of examples.

Stored and forward mechanism has been proposed (Fall, 2003; Cerf et al., 2004) to deliver messages in such challenging communication environments. Messages are sent from one node to another, stored at that node for a while before being forwarded again when opportunities arise. The challenges of designing efficient DTN routing protocols are

\section{- to determine which node to forward}

- when to forward.

Several DTN routing algorithms have been proposed (Jain et al., 2004; Burgess et al., 2006; Wang et al., 2005; Zhao et al., 2004; Spyropoulos et al., 2005). The authors in Jain et al. (2004) design four knowledge-based routing schemes. Their schemes assume that certain information about the networks such as apriori knowledge of link connectivity patterns, geographical locations, or the node movement schedules are available. Routing decisions are made using Dijkstra-like algorithms to decide when and how a message should be forwarded. The four schemes are Earliest Delivery (ED), Minimum Expected Delay (MED), Earliest Delivery with Local Queue (EDLQ), and Earliest Delivery with All Queues (EDAQ) schemes. The ED scheme assumes that aggregate statistics of contacts (a contact is an encounter between two nodes that provides data transfer opportunity) is known. However, aggregate statistics of contacts may not be available apriori. The approach in Burgess et al. (2006) and Lindgren et al. (2003) belongs to another category of DTN routing where probabilistic metrics are used to keep contact history. Such metrics are used to determine delivery probabilities to any destination node via an intermediate node. The delivery probability ages with time and also has a transitive property. Message ferrying approach (Zhao et al., 2004) has also been proposed for DTN routing. Message ferrying approach is especially useful when the network is so sparse that some nodes may not come into contact with one another at all. In Spyropoulos et al. (2005), the authors propose Spray and Wait where L copies of the messages are sent by the source to different contacts and these messages are stored by the contacts and delivered only if one of the contacts meets the destination node. Hence, a message will be delivered in only two hops. However, such 2-hop routing approach does not work when the contacts cannot meet the destination node. Coding-based approach (Wang et al., 2005; Jain et al., 2005) has also been proposed. The basic idea of the erasure coding approach is to encode an original message into a number of coding blocks, say $\mathrm{n}$, and a destination only needs to receive $\mathrm{k}$ out of these $\mathrm{n}$ coding blocks to be able to reconstruct the original message. The different blocks are transmitted by the sender to different nodes that the sender encounters. A two hop routing approach is used for the erasure-coding approach which means the source node selects $\mathrm{n}$ relay nodes and these relaying nodes that receive such blocks can only forward them to the destination. A summary of some 
existing routing approaches for DTNs can be found in Zhang and Zhang (2007)

None of the above approaches deal with multicast traffic delivery in DTN environments. Existing multicast routing approaches for Mobile Ad Hoc Networks (MANETs) cannot be applied directly to DTN environments because it is difficult to maintain a multicast tree or mesh structure during the lifetime of a multicast session due to the network sparseness and node mobility. In addition, data transmissions would suffer from many failures due to the intermittent connections. The network may not be connected at all. Zhao et al. (2005) is the first paper that discusses multicast delivery in DTN. The authors in Zhao et al. (2005) propose new semantic models for DTN multicast and develop several multicast routing algorithms. For example, the authors describe a Temporal Membership (TM) model where a membership interval is associated with each message. This membership interval specifies the period during which the group members are defined. Additional refinement for the TM model includes a delivery interval which defines the time period during which the message should be delivered to the intended receivers. The routing schemes proposed in Zhao et al. (2005) include

- unicast-based routing where as many messages as the number of intended receivers are created and each message is delivered via a unicast routing scheme to a receiver

- $\quad$ tree-based routing where messages are forwarded along a tree in the DTN graph that is rooted at the source and reaches all receivers

- group-based routing where a forwarding group is responsible for forwarding the message.

Messages will be flooded within the forwarding group to increase the chance of delivery. The results presented in Zhao et al. (2005) indicate that the DTBR scheme which dynamically constructs the multicast delivery tree gives the best performance. However, the authors in Ye et al. (2006) observe that DTBR makes early decision to subdivide the receiver list at a branching point and hence may prevent multicast packets from being delivered to a receiver using an alternative path from another tree branch. Such a weakness prevents DTBR from achieving high delivery ratio in some scenarios. Thus, the authors in Ye et al. (2006) propose an improved algorithm called on-demand situational multicast (OS-Multicast) scheme that can achieve a higher delivery ratio but at the cost of lower delivery efficiency. We will elaborate more on both DTBR and OS-Multicast schemes in Section 4.

\section{System models}

\subsection{Network model}

A DTN is an overlay network that is built upon underlying networks e.g., wireless ad hoc networks. Its network architecture is based on the asynchronous message (called bundle) forwarding paradigm presented in Fall (2003). Only those nodes that implement the DTN functionalities e.g., sending, storing, and receiving bundles are considered DTN nodes, while the others are denoted as normal nodes. A DTN link may span several underlying links. Figure 1 depicts a simple DTN example. In Figure $1, n_{3}$ and $n_{4}$ are neighbours in the DTN layer but in the underlying network layer, this DTN link goes through two wireless hops (via $n_{6}$ and $n_{7}$ ). A DTN node $j$ is considered a neighbour of another DTN node $i$ only if there is an end-to-end path connecting them in the underlying network. In this work, we assume the underlying network is ad hoc network and hence an ad hoc networking routing scheme like DSR (Broch et al., 1998) is used to find a route between any two DTN nodes.

\section{Figure 1 A simple DTN example}

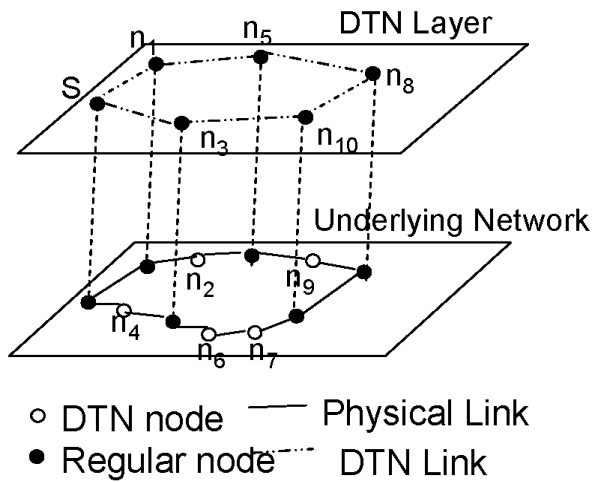

In the DTN layer, bundles are transmitted in a store-and-forward manner hop by hop. This means that when a DTN node has unicast/multicast bundles to deliver, DTN unicast/multicast routing scheme will be used. If a DTN node cannot find an available next-hop DTN node to deliver the bundles, then the bundles will be stored in its buffers. Each DTN node has finite-size buffers. To ensure reliable delivery, a custodian transfer feature has been proposed (Fall et al., 2003). When the custody transfer feature is turned on, a DTN node $i$ will find another DTN node $j$ which is willing to accept the custody of a particular bundle i.e. the other node is willing to accept the responsibility of delivering the bundle to its destination. Node $i$ will send node $j$ a custody request message and if node $j$ is willing, node $j$ will reply with a custody accept. The custody request message is piggybacked to the bundle that node $i$ sends node $j$. Once node i receives such an acknowledgement, it can remove the sent bundle from its buffers.

\subsection{Multicasting model}

Multicast in DTNs is defined as the one-to-many or many-to-many bundle transmissions among a group of DTN nodes. A multicast source uses either a multicast End-point Identifier Descriptor (EID) e.g. *.cse.lehigh.edu, or an explicit list of the names of individual DTN multicast receivers as the destination address for multicast bundles. 
The later approach may not be scalable when the number of DTN multicast receivers grow large.

\section{Group membership management}

Several semantic models of DTN multicast membership have been studied in Zhao et al. (2005). For example, in the Temporal Membership (TM) semantic model, each bundle includes a membership interval that specifies the period during which the group members are defined. If the membership interval of a bundle from a multicast group $G$ is $[t 1, t 2]$, it means that the intended receivers of the message consist of all members of the multicast group $G$ at any time during $[t 1, t 2]$. Multicast messages can be delivered at any time. We adopt this TM model for our multicast work.

A DTN node $k$ can send the multicast source a Group_Join message with the start-time $t s_{k}$ and end-time $t e_{k}$. The multicast source can add this node's membership information into a receiver list that it maintains. The membership information can be included in the bundles to be delivered or sent separately as control messages to next-hop custodians. The inclusion of membership information in the bundles works for small groups but separate control messages have to be used for large groups. The membership information allows each intermediate custodian, $m$, to check if the membership of a receiver, $v$, has expired. If a multicast bundle carries a list of valid receivers in its header, then node $m$ will not include $v$ 's identifier in the relayed bundle if $v$ 's membership expires. If a multicast EID is used, then node $m$ updates the information it maintains when it receives new membership information, and relays the bundle only if there is at least one active downstream receiver (i.e., a receiver whose membership has not expired).

\section{Multicasting routing approaches for DTNS}

\subsection{Existing multicasting approaches}

As we mentioned before, DTNs suffer from frequent network partitions. The dynamically changing network topology makes the maintenance of any multicast tree in DTNs challenging. The performances of different multicast approaches depend on how much current network information is available to the DTN nodes. Therefore, situational information needs to be collected and disseminated continuously so that new information can be used to adjust the message delivery paths. Such data collection and dissemination procedures incur overhead. Thus, a tradeoff between improving delivery performance and incurring extra overhead needs to be done. Various policies that utilise different amounts of situational information for routing decisions can be created for applications with varying quality of service requirements.

An obvious multicast delivery approach is to let the source send a separate bundle to each of the receivers. Each duplicated bundle will be delivered to its intended receiver using the DTN unicast routing scheme. This is referred to as U-Multicast. This approach is wasteful since a DTN node may receive multiple copies of the message intended for different receivers. In addition, there are two existing approaches for supporting multicast communications in a DTN, namely DTBR and OS-Multicast. An illustrative example of these two approaches is shown in Figure 2. Note that the nodes shown in the Figure 2 are DTN nodes so nodes 1 and 3 may not be direct neighbours in the underlying network. We assume in this work that the underlying network is ad hoc network and hence an adhoc networking routing scheme like DSR is needed to find a route between any two DTN nodes.

- $\quad \operatorname{DTBR}$ (Zhao et al., 2005): This is a dynamic tree-based multicasting algorithm designed for DTNs. In DTBR, the upstream node will assign the receiver list for its downstream neighbors based on its local view of the network conditions. The downstream nodes are required to forward bundles only to the receivers in the list, even if a new path to another receiver (not in the list) is discovered. For example, in Figure 2(a), let us assume that node 1 decides to use node 2 to deliver to nodes 4,5 , and node 3 to deliver to node 6 . Assume that when the bundle reaches node 2, link 2-5 becomes unavailable but link 3-5 is available. DTBR will not be able to utilise this opportunity since the header for the bundle received at node 3 only indicates that node 6 is the receiver. DTBR also assumes that each node has complete knowledge or the summary of the DTN link states in the network. However, this is hard to achieve in practical scenarios.

- On-demand Situation-aware multicast (OS-multicast) (Ye et al., 2006) Like the DTBR scheme, OS-multicast is also a dynamic tree-based multicast approach. A unique multicast tree is constructed for each bundle and the tree is adjusted at each intermediate DTN node according to the current network conditions. When a DTN node receives a bundle, it will dynamically adjust an initially constructed tree based on its current knowledge of the network conditions. Via such adjustments, any newly discovered path will be quickly utilised. For example, in Figure 2(b), the link between 2 and 5 is broken but when the bundle reaches node 3 , node 3 knows that it can reach node 5 and 6 . So, it will send a copy to both nodes 5 and 6 . The downside of the OS-multicast approach is that a receiver may receive multiple copies of the same bundle. 
Figure 2 Multicast approaches in DTN: (a) DTBR and (b) OS-multicast: when link $2 \rightarrow 5$ is unavailable and link 3 to 5 becomes available, node 3 will take advantage of the current available link immediately

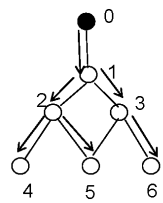

(a)

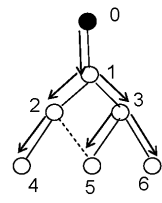

(b)

\subsection{Context Aware Multicast Routing (CAMR) scheme}

In this section, we describe the CAMR scheme we proposed. The CAMR scheme consists of five components:

- local node density estimation

- 2-hop neighbour contact probability estimate

- route discovery

- route repair

- data delivery.

The pseudo codes for the CAMR scheme are shown in Figure 3.

Figure 3(a) Local node density estimation

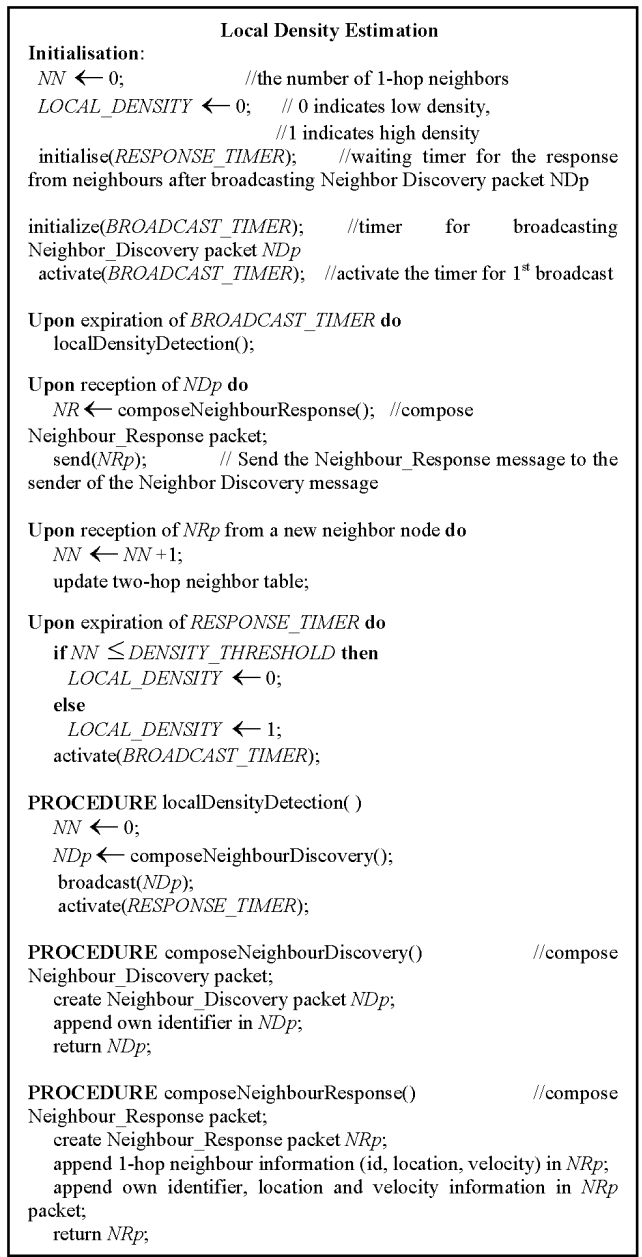

Figure 3(b) Two-hop neighbour contact estimation

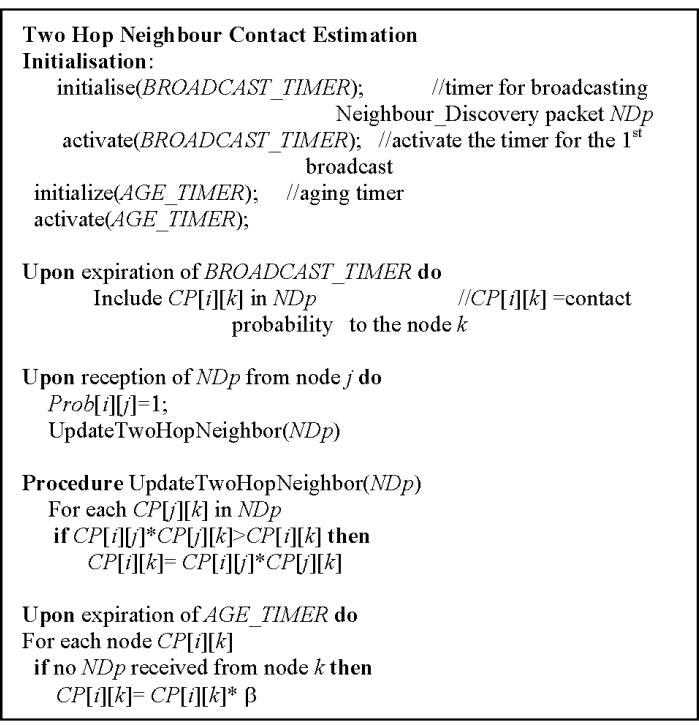

Figure 3(c) Pseudo code for route discovery

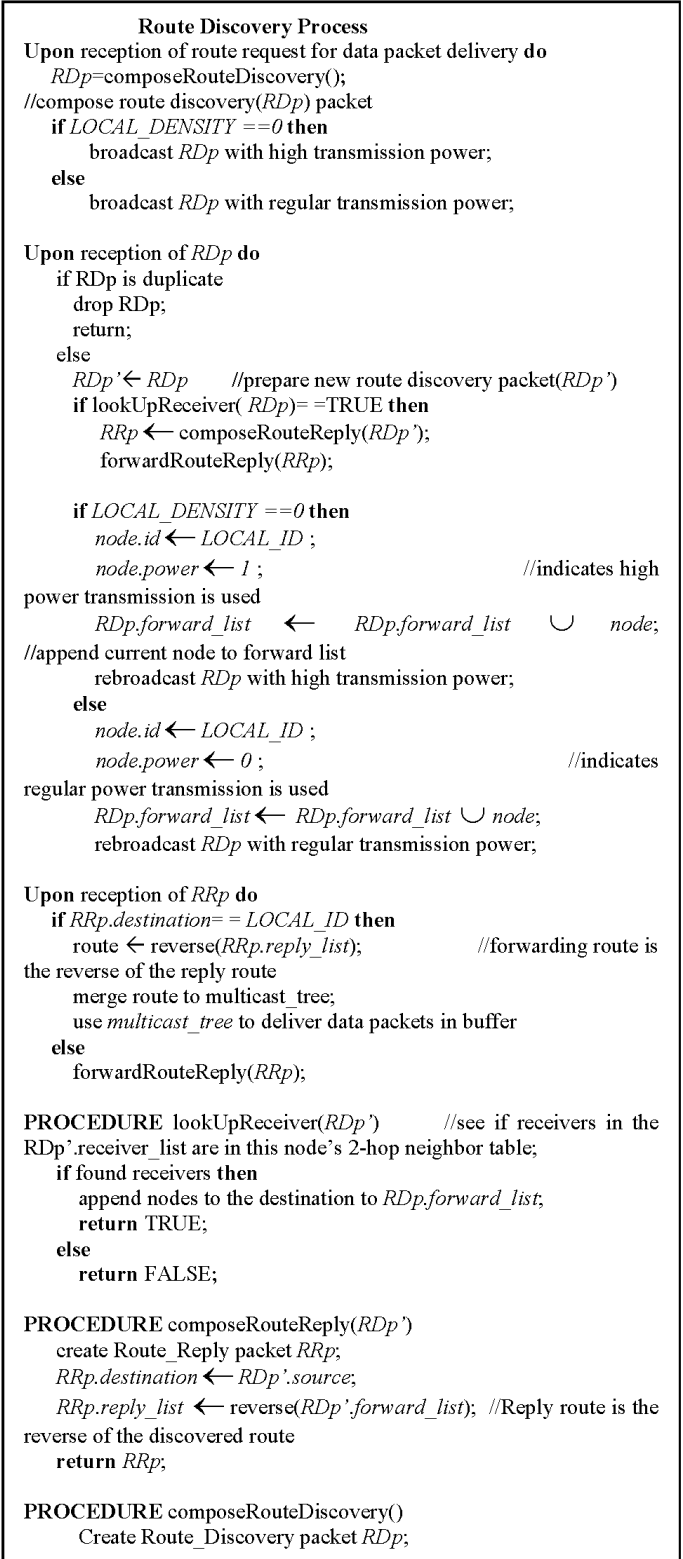


Figure 3(d) Pseudo code for route repair

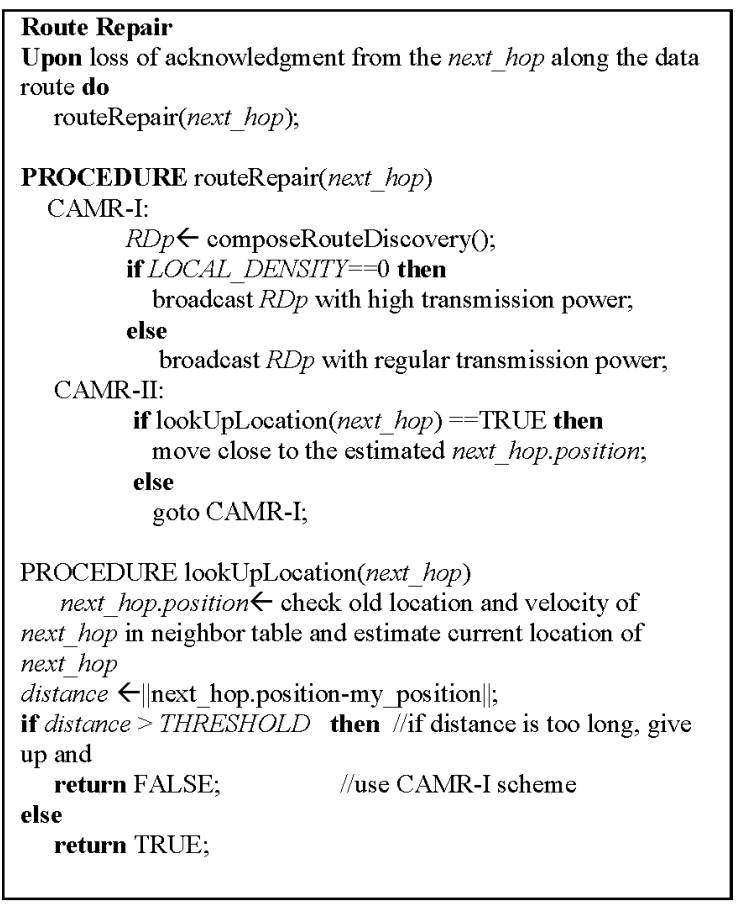

\section{Local node density estimation}

Each node periodically (e.g. every $20 \mathrm{~ms}$ ) broadcasts a neighbour discovery packet using regular power transmission. On receiving a neighbour discovery packet, a node composes a neighbour response packet including this node's information (e.g., identifier, location, and velocity) and this node's 1-hop neighbour's information (e.g., the neighbour's identifier, location, and velocity) and sends the neighbour response packet to the originator of the a neighbour discovery packet after some random backoff delay. Thus, each node can estimate the number of neighbours it has periodically, denoted as $N_{d}$. If a node's $N_{d}$ drops below a threshold, $K$, during a neighbour discovery period, the node sets a sparsely connected flag. Figure 3(a) shows the pseudo code for the local node density estimation.

\section{2-hop neighbour contact estimation}

Each node also maintains its contact probabilities with its 2-hop neighbours. The contact probability of a neighbour is set to 1 as long as a node, $n_{i}$, can receive neighbour response messages from a neighbour, $n_{i}$, periodically. When $n_{i}$ fails to hear a neighbour response message from $n_{j}$, then $n_{i}$ decreases its contact probability with $n_{j}$ by a factor of $\beta$ periodically (since the neighbour discovery message is sent out periodically). Rather than immediately reducing the contact probability to zero, the aging factor, $\beta$, is used to avoid the ping-pong effect (i.e. a node may move in and out of the Transmission Range (TR) frequently). These contact probabilities allow a node to send the messages directly without incurring the route discovery overhead if the destination happens to be within its 2-hop neighbourhood.

\section{Route discovery}

A source initiates a route discovery process if it cannot reach any of the receivers to which the multicast traffic needs to be delivered. Before any intermediate node re-broadcasts a route request message it receives, it first checks to see if its sparsely connected flag is set. If the flag is set, then the node rebroadcasts the route request message using high power transmission (e.g., at a level that results in a TR of $375 \mathrm{~m}$ or $500 \mathrm{~m}$ ). Otherwise, the intermediate node re-broadcasts the route request using regular power. Any node that receives a high power route request will make a note since that node needs to issue a high power route reply when it hears a response back from a downstream node.

Assume that $S$ is the multicast source and there are eight multicast receivers as shown in Figure 4. $S$ knows it can reach $R_{6}$ using its 2-hop neighbourhood information so $S$ does not need to issue any route request for $R_{6}$. The route request issued by $S$ for the other seven receivers is flooded by intermediate nodes using regular power until it reaches node $R_{6}$ and $n_{3} . R_{6}$ and $n_{3}$ will re-broadcast the route request using high power since their observed local node densities drop below the threshold $K$. When the route request eventually reaches any intended multicast receiver, it will issue a route reply. Through this process, $S$ can eventually construct a merged multicast delivery tree after hearing route replies from downstream nodes for all the eight receivers. Note that this multicast tree is not static as in wired multicast or static wireless mesh network scenario. Note further that the location and velocity information of the nodes sending route request and route reply messages are piggybacked. For example, when $R_{6}$ receives a route reply from $n_{4}, R_{6}$ will record the location and velocity information of $n_{4}$ and that $n_{4}$ is the next hop node for delivering bundles from this multicast session. Later, when $S$ sends multicast bundles, $S$ will piggyback a multicast receiver list in all multicast bundles that are sent out. Thus, $R_{6}$ knows that it is responsible for delivering the multicast messages to $R_{1}, R_{5}, R_{7}$ and $R_{8}$. Upon receiving the multicast bundles, $R_{6}$ travels closer to $n_{4}$ so that the multicast bundles can be delivered using regular power transmission. Since the location and velocity information is included in the route reply from $n_{4}$ to $R_{6}, R_{6}$ can estimate where it can reach $n_{4}$ with regular power transmission. Note that $R_{6}$ may decide to travel towards $n_{4}$ only after receiving multiple multicast bundles (aka batch delivery). Similar actions are taken by $n_{3}$ to deliver multicast bundles to $n_{6}$.

Figure 3(c) shows the pseudo code for our route discovery process. There are several optimisations that one can make e.g., the source can flood a multicast route request where the identifiers of all receivers are included rather than sending individual route request, the intermediate nodes can merge the route replies from different downstream nodes that can reach different multicast receivers. In this paper, our simulation study does not include these optimisations. 
Figure 4 CAMR scheme

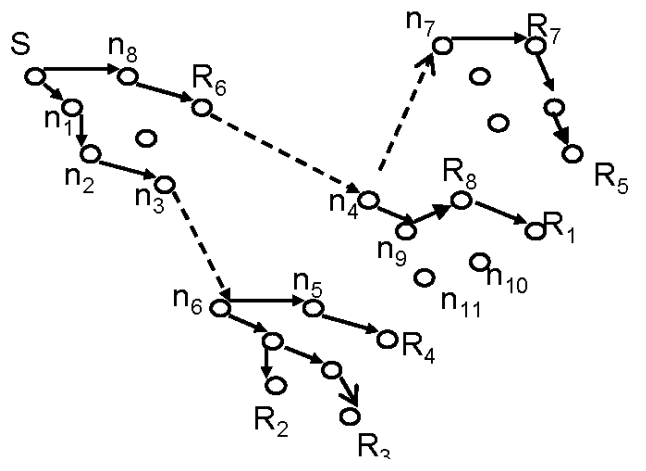

\section{Route repair}

In CAMR, one can use local route repairs when the multicast tree is broken as a result of node mobility. Let us assume that when multicast bundles arrive at node $n_{4}$ in Figure $4, n_{9}$ moves away. There are two ways whereby $n_{4}$ can repair the route:

- $\quad n_{4}$ can issue a route request to find a route to $R_{8}$

- $\quad n_{4}$ can make use of the location and velocity information to travel closer to $n_{9}$ and not incur extra route discovery messages for local repair.

We refer to the version where $n_{4}$ issues a route request message to perform the local route repair as CAMR-I and the version where $n_{4}$ uses the location and velocity information to perform route repair as CAMR-II. The CAMR-II scheme will incur smaller routing overhead than the CAMR-I scheme. Figure 3(d) shows the pseudo code for our route repair procedure.

\section{Data delivery}

For data delivery, an extra header is piggybacked to each data bundle. The header contains information on the identifiers of the receivers to which a particular multicast bundle needs to be delivered. Any intermediate node that supports CAMR scheme will duplicate the bundle if the node discovers that it is the branching point. A node acts as a message ferry during data delivery if it uses a high power route request to reach a downstream node. Such a node will only move as a message ferry after accumulating enough bundles (the threshold is denoted as MOVING_THRESHOLD). The ferrying node travels towards the last known position of the next-hop DTN node. If the ferrying node hears the beacons from this next-hop DTN node, then it can transfer all the accumulated bundles destined for this next hop node. Since the down-stream next hop node may have already moved by the time a ferrying node moves, this ferrying node may not find that next-hop node. In that case, this ferrying node will return to its original position and perform a route rediscovery for the receivers that it needs to reach. Custodian transfer feature (Fall et al., 2003) is turned on in our CAMR scheme. This means that after a node $n_{i}$ receives a custodian acknowledgment from a downstream node, $n_{j}, n_{i}$ can remove the acknowledged bundle from its storage.

\section{Performance evaluation}

\subsection{Simulation set up}

To evaluate the performance of different multicast algorithms, we implemented U-multicast, OS-multicast, DTBR, and CAMR (only version II) in the $n s 2$ simulator (UCB/LBNL/INT). The performance metrics that are used to compare different multicast routing approaches are:

- message Delivery Ratio (DR), which is defined as the number of unique multicast packets which successfully arrive at all the receivers over the total number of packets which are expected to be received, e.g. if the source sends out $N$ packets with $R$ receivers and each receiver receives $x_{i}$ packets, then $D R=\sum x_{i} / N \times R$

- data efficiency, which is the ratio between the number of unique multicast packets successfully delivered to the receivers, and the total data traffic generated in the networks

- overall efficiency, which is the ratio between the number of unique multicast packets successfully delivered to the receivers, and the total traffic generated (both data and control packets) in the networks

- average message delay, which is the average of the end-to-end bundle delivery latencies for each algorithm (we observe similar delay performance results when we use the metric of median delay).

Note that for the overall efficiency computation, we assume that the power required to transmit a data packet is a linear function of the packet size. In addition, we assume that a route request or route reply message is 35 bytes long. Each route request or reply message that is transmitted at high power is counted as $\left(k=\left(r_{2} / r_{1}\right)^{4}\right)$ times that of a route request/reply message that is transmitted at regular power where $r_{2}$ and $r_{1}$ is the TR of the high and regular power transmission respectively e.g., $k=16$ when the high power TR is $500 \mathrm{~m}$ but the regular power TR is $250 \mathrm{~m}$. The neighbour discovery interval is set at $10 \mathrm{~s}$, and $\beta$ is set at 0.8 in all our experiments unless otherwise stated.

In our experiments, unless otherwise stated, we use a network with 40 nodes deployed randomly in a geographical area of size $4000 \times 4000 \mathrm{~m}^{2}$. All nodes are DTN nodes unless otherwise stated. DSR is used as the routing approach for the underlying ad hoc networks for U-Multicast, DTBR and OS-Multicast schemes. And situational awareness is achieved through the communication between the DTN multicasting agent and the DSR routing agent. For CAMR, unless otherwise stated, the speed of the nodes not acting as message ferry is chosen uniformly between $1 \mathrm{~m} / \mathrm{s}$ and $5 \mathrm{~m} / \mathrm{s}$. When a node acts as a message ferry, it moves with a speed of $15 \mathrm{~m} / \mathrm{s}$. The MAC layer is IEEE 802.11 with radio TR that varies from $250 \mathrm{~m}$ to $500 \mathrm{~m}$. Table 1 summarises the default simulation parameter values used. For each simulation run, we generate traffic for $1000 \mathrm{~s}$ and run the simulation for $10,000 \mathrm{~s}$. Each data point is the average results obtained from at least five runs. 
Table $1 \quad$ Simulation parameters

\begin{tabular}{ll}
\hline Parameter & Value \\
\hline Simulation area & $\begin{array}{l}1000 \times 1000,2000 \times 2000,3000 \times 3000, \\
4000 \times 4000 \mathrm{~m}^{2}\end{array}$ \\
Simulation time & $10,000 \mathrm{~s}$ \\
Buffer size & 600 messages \\
ND Interval/ & $10 \mathrm{~s} / 0.8$ \\
High power TR & Default: $500 \mathrm{~m}$ \\
Moving threshold & 10 \\
(CAMR) & \\
Traffic pattern & Default: 1 group 12 receivers \\
& $\begin{array}{l}\text { Multicast source generates CBR traffic } \\
\text { with a packet size of } 512 \text { byte }\end{array}$ \\
Mobility model & $\begin{array}{l}\text { Default: RWP with }\left(v_{\text {min }}=1 \mathrm{~m} / \mathrm{s},\right. \\
\left.v_{\max }=5 \mathrm{~m} / \mathrm{s}\right)\end{array}$ \\
& Others: Zebranet, UMassBusNet \\
\hline
\end{tabular}

\section{Mobility models}

In this work, we use three mobility models, namely

- $\quad$ Random Waypoint (RWP) model

- Zebranet (Wang et al., 2005) model

- UMassBusNet (Zhang et al., 2007) model.

In the RWP model, each node selects a randomly located destination and move towards that destination with constant speed. Once it reaches the destination, it pauses for a certain period of time before repeating the process again. The speed of the nodes not acting as message ferry is chosen uniformly between $1 \mathrm{~m} / \mathrm{s}$ and $5 \mathrm{~m} / \mathrm{s}$. For the Zebranet model, we create a semi-synthetic Zebranet mobility model as follows: we synthesise node speed and turn angle distributions from the observed data and create other node-movements using the same distribution. We use both distance and time scaling to fit the original data found in the trace into the network environment that we are interested in. For the UMassBusNet model, we extract the locations of 20 buses at different times in one trace, and scale their relative locations to fit into the geographical area of interest.

We first evaluate the sensitivity of the delivery performance of our CAMR scheme when certain tunable parameters e.g., high power TR, $\beta$ are varied. Then, we compare the CAMR scheme with other DTN multicast schemes e.g. U-Multicast, DTBR (Zhao et al., 2005) and DTBR (Ye et al., 2006). Next, we study the delivery performance of CAMR in different scenarios e.g., varying number of groups, varying group size, different mobility models.

\subsection{Sensitivity analysis of parameters in CAMR}

\subsubsection{Impact of transmission range}

First, we evaluate the difference in the delivery performance when the high power TR is set either to $375 \mathrm{~m}$ or $500 \mathrm{~m}$. We use one multicast group with one source and 12 receivers. The multicast source generates traffic at a rate of $1 \mathrm{pkt} / \mathrm{s}$. Tables 2 tabulate the delivery ratio, average delay, and the data efficiency results that we obtain when the high power TR is set to either $375 \mathrm{~m}$ or $500 \mathrm{~m}$ respectively. The delivery ratio achieved using a high power TR of $500 \mathrm{~m}$ is slightly better than using $375 \mathrm{~m}$. The average delay with $500 \mathrm{~m}$ is also smaller. Thus, we use $500 \mathrm{~m}$ as the high power TR for the rest of our study in this paper.

Table 2 Results using high power Tx range of 375/500 m

\begin{tabular}{ccccccc}
\hline & \multicolumn{3}{c}{$\begin{array}{c}\text { Average } \\
\text { delay }(\mathrm{s})\end{array}$} & \multicolumn{2}{c}{$\begin{array}{c}\text { Data efficiency and } \\
\text { overall efficiency }\end{array}$} \\
\cline { 2 - 7 } Area $\left(\mathrm{m}^{2}\right)$ & $375 \mathrm{~m}$ & $500 \mathrm{~m}$ & $375 \mathrm{~m}$ & $500 \mathrm{~m}$ & $375 \mathrm{~m}$ & $500 \mathrm{~m}$ \\
\hline $1000 \times 1000$ & 0.93 & 0.93 & 81 & 81 & $0.40 / 0.18$ & $0.40 / 0.18$ \\
$2000 \times 2000$ & 0.89 & 0.90 & 2421 & 2327 & $0.37 / 0.16$ & $0.36 / 0.14$ \\
$3000 \times 3000$ & 0.82 & 0.85 & 2845 & 2700 & $0.31 / 0.14$ & $0.31 / 0.11$ \\
$4000 \times 4000$ & 0.75 & 0.80 & 3953 & 3725 & $0.28 / 0.12$ & $0.27 / 0.10$ \\
\hline
\end{tabular}

\subsubsection{Impact of different Neighbor Discovery interval and aging factor values}

We conduct a set of experiments to investigate the impact of using different values for the Neighbor Discovery (ND) interval with the 40 nodes over $4000 \times 4000 \mathrm{~m}^{2}$ network scenario. We use a single multicast group with 12 receivers and the multicast source generates packets at rate of $1 \mathrm{pkt} / \mathrm{s}$. The results are tabulated in Table 3(a). With an interval of $10 \mathrm{~s}$, the delivery ratio is $80 \%$ but with $20 \mathrm{~s}$, it drops to $74 \%$. When the ND interval is longer, the nodes make routing decisions based on older information and hence the delivery performance suffers. We will use a ND interval of $10 \mathrm{~s}$ for the rest of our paper.

We also conduct a study on the impact of different aging factors on the data delivery performance. The results are tabulated in Table 3(b). The paired $t$-tests (Navidi, 2006) show that the difference in the observed results is not statistically significant. This means that the delivery performance remains relatively the same when the $\beta$ value lies within the range $(0.4,0.8)$. Thus, we decide to use $\beta=0.8$ for the rest of our paper.

Table 3(a) Results with different Neighbour Discovery intervals

\begin{tabular}{lcccc}
\hline $\begin{array}{l}\text { ND } \\
\text { interval (s) }\end{array}$ & $\begin{array}{c}\text { Delivery } \\
\text { ratio }\end{array}$ & $\begin{array}{c}\text { Average } \\
\text { delay (s) }\end{array}$ & $\begin{array}{c}\text { Data } \\
\text { efficiency }\end{array}$ & $\begin{array}{c}\text { Overall } \\
\text { efficiency }\end{array}$ \\
\hline 10 & 0.80 & 3725 & 0.27 & 0.1 \\
15 & 0.76 & 3852 & 0.26 & 0.1 \\
20 & 0.74 & 4122 & 0.28 & 0.1 \\
\hline
\end{tabular}

Table 3(b) Results with different aging factors

\begin{tabular}{lcccc}
\hline $\begin{array}{l}\text { Aging } \\
\text { factor }\end{array}$ & $\begin{array}{c}\text { Delivery } \\
\text { ratio }\end{array}$ & $\begin{array}{c}\text { Average } \\
\text { delay }(\mathrm{s})\end{array}$ & $\begin{array}{c}\text { Data } \\
\text { efficiency }\end{array}$ & $\begin{array}{c}\text { Overall } \\
\text { efficiency }\end{array}$ \\
\hline 0.4 & 0.83 & 3628 & 0.27 & 0.1 \\
0.6 & 0.81 & 3673 & 0.27 & 0.1 \\
0.8 & 0.81 & 3725 & 0.27 & 0.1 \\
\hline
\end{tabular}




\subsection{Comparison with other multicast routing approaches}

In this section, we compare the CAMR scheme with U-Multicast, DTBR and OS-Multicast schemes using the random waypoint mobility model with 40 nodes distributed in an area which ranges from $1000 \times 1000 \mathrm{~m}^{2}$ to $4000 \times 4000 \mathrm{~m}^{2}$. A single multicast group with 12 receivers is used in this set of experiments. The multicast source generates packets at a rate of $0.5 \mathrm{pkt} / \mathrm{s}$. Figure 5(a)-(c) plot the delivery ratio, the average delay and the data efficiencies of the different schemes.

Figure 5 (a) Delivery ratio using RWP mobility model; (b) average delay using RWP mobility model and

(c) data efficiency using RWP mobility model

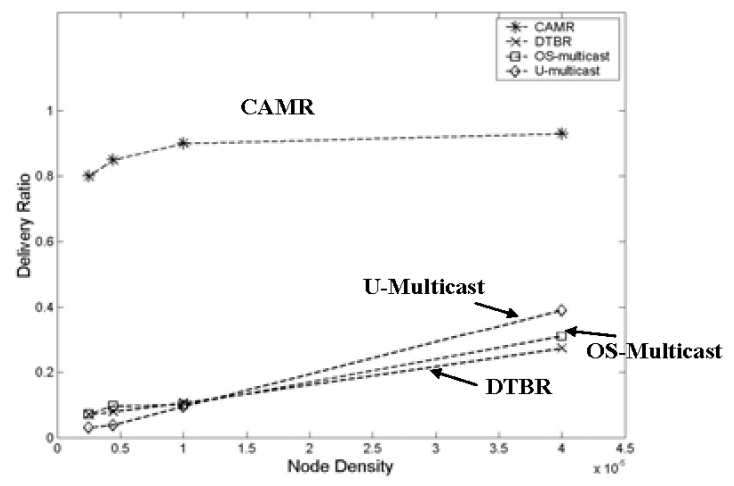

(a)

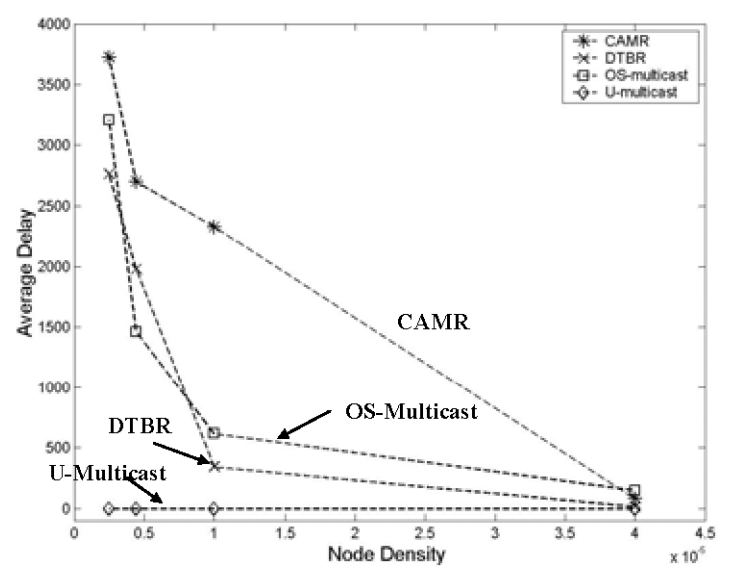

(b)

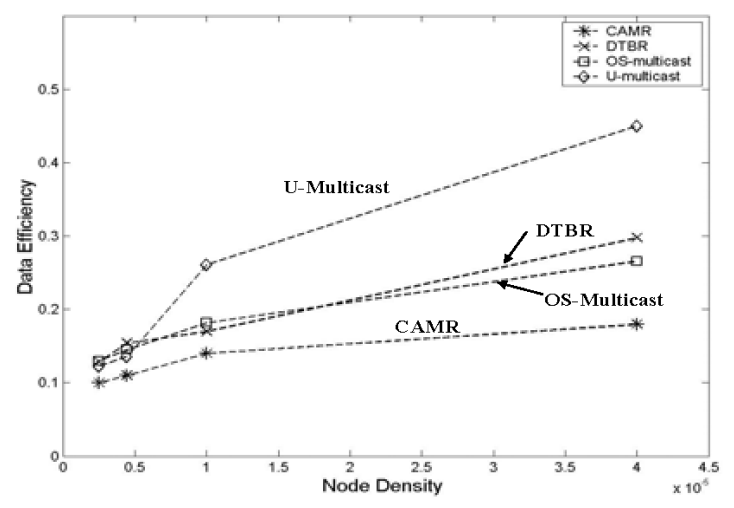

(c)
From the plots, we see that CAMR achieves the highest delivery ratio (see Figure 5(a)) with similar delay performance. The price to pay is a slight decrease in data efficiency (see Figure 5(c)). The average delay in CAMR may look larger (see Figure 5(b)) because of a longer tail in the delivery latency distribution. However, if we consider only those packets that are successfully delivered in all schemes, the average delay achieved by CAMR is smaller e.g., DTBR can only achieve $9.7 \%$ delivery ratio in the 40 nodes by $3000 \times 3000 \mathrm{~m}^{2}$ scenario and has an average delay of $1460 \mathrm{~s}$ and a data efficiency of 0.145 . Using CAMR, the average delay of similar packets is about $129 \mathrm{~s}$ with a data efficiency of 0.39 .

We expect that the CAMR scheme will outperform all other schemes when the network becomes very sparse. Since store-and-forward approach is not used for real time traffic but to ensure that many messages can be delivered even in scenarios where no end-to-end paths exist, we believe that our CAMR scheme is the best choice for multicast delivery especially when the networks are very sparse e.g. with only 0.5 neighbour within its TR.

\subsection{Impact of mobility models}

In this section, we investigate the impact of mobility models on the delivery performance of the CAMR scheme. We use the default network scenario with 40 nodes distributed over $4000 \times 4000 \mathrm{~m}^{2}$. The nodes move according to either the

- RWP

- Zebranet

- UMassBusNet model.

We use a multicast group with 12 receivers. The packet rate generated by the multicast source is varied from $0.25 \mathrm{pkt} / \mathrm{s}$ to $2 \mathrm{pkt} / \mathrm{s}$. Figure 6(a)-(c) plot the delivery ratio, the average delay, and the data efficiency results that we obtain.

From the plots, we can draw a few conclusions. Among the three mobility models, the UMassBusNet model results in the poorest delivery performance. The paired t-test results did show that the difference in the delivery ratio between RWP/Zebranet and UMassBusNet is significant (with a $\mathrm{P}$ value $<0.008$ ). The poorer delivery performance in the UMassBusNet model is due to two factors, namely

- The mean intercontact time in UMsasBusNet model is longer and its mean contact duration is shorter. This means fewer packets can be transferred when two nodes encounter each other and that packets need to be queued longer and hence have high probability of being dropped as a result of buffer overflows

- The nodes move in different clusters. Those nodes (buses) that move using similar routes are in one cluster and packets transferred between these nodes have better delivery performance. When nodes are in different clusters, it is difficult to find a relaying node that can help to transfer packets between these two clusters. 
Figure 6 (a) Delivery ratio vs. packet rate; (b) average delay vs. packet rate and (c) data efficiency vs. packet rate

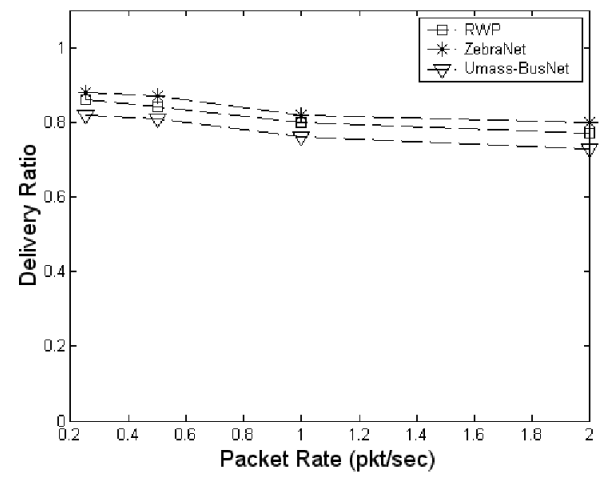

(a)

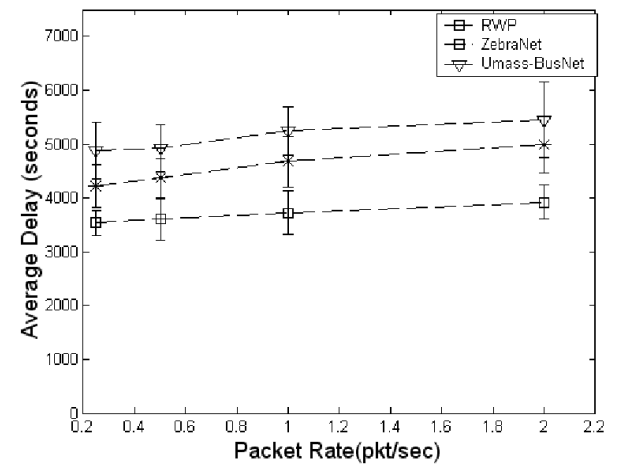

(b)

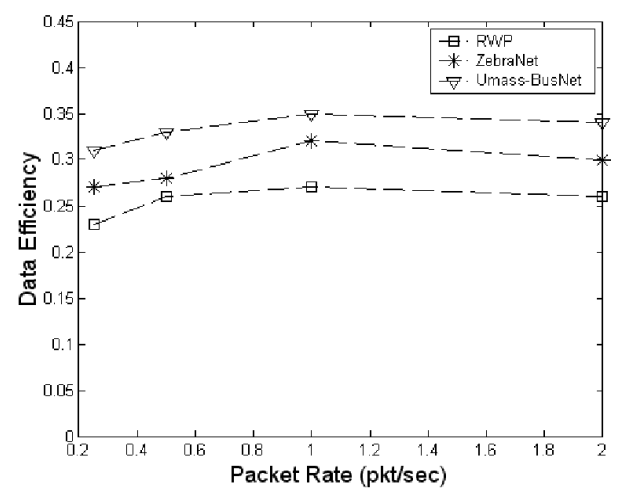

(c)

The delivery performance using the Zebranet model is better than that using the RWP model because the nodes in the Zebranet model move faster (the average node speed is $6 \mathrm{~m} / \mathrm{s}$ in Zebranet model but only $3 \mathrm{~m} / \mathrm{s}$ in the RWP model). The mean intercontact time and the contact duration between any two nodes are shorter in the Zebranet model. However, the contact duration in the Zebranet model is long enough to allow nodes to transfer queued packets to other nodes during their encounters. With shorter intercontact time, packets will be queued shorter. Thus, the delivery ratio is higher in the Zebranet model.

The data efficiency is the best using the UMassBusNet model because the average number of hops of successfully delivered packets in this model is 2.1. Those packets that require more hops are dropped. The average number of hops taken to deliver packets using the Zebranet model is 2.6 (faster node speed in the Zebranet model results in shorter path) while that for the RWP model is 3 . That explains why the data efficiency for the Zebranet model is higher than that for the RWP model.

\subsection{Impact of different number of multicast groups}

In this section, we investigate the impact of different number of multicast groups on the delivery performance of the CAMR scheme. We use the default network scenario with 40 nodes distributed over the $4000 \times 4000 \mathrm{~m}^{2}$. The nodes are allowed to move according to either the RWP or the UMassBusNet models. Each group has six receivers randomly chosen from the 40 nodes. Each multicast source generates packets at a rate of $1 \mathrm{pkt} / \mathrm{s}$. We vary the number of multicast groups from one to six. Figure 7(a)-(c) plots the delivery ratio, the average delay and the data efficiency results that we obtain.

Figure 7 (a) Delivery ratio vs. No. of groups; (b) average delay vs. No. of groups and (c) data efficiency vs. No. of groups

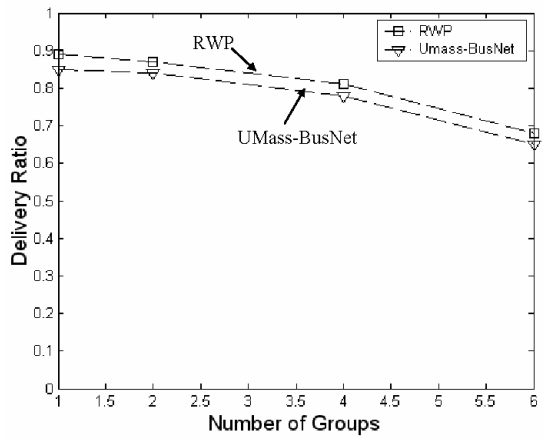

(a)

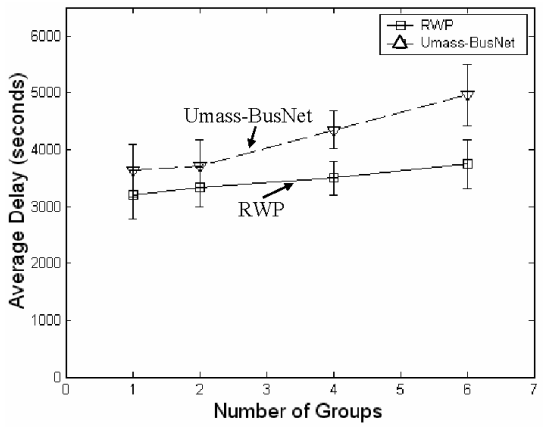

(b)

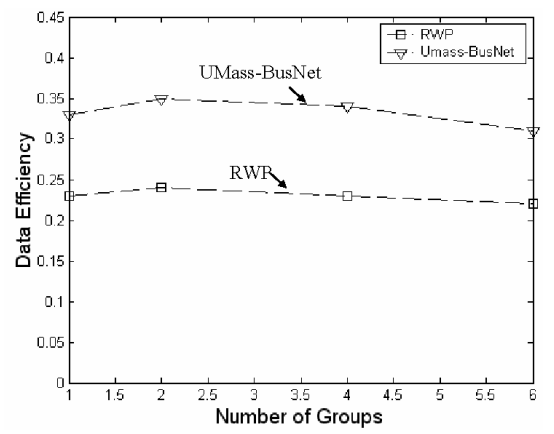

(c) 
There are several observations we made from the results. First, the delivery performance of the CAMR scheme degrades slowly with increasing number of groups and hence it shows that the scheme is scalable. Second, as the number of groups increases beyond four groups, the delivery ratio degrades faster. With both mobility models, the delivery ratio drops only by $8-10 \%$ as the number of groups increases from one to four but drops by $16 \%$ as the number of groups increases from four to six. Such degradation is due to buffer overflows since the total packets each node needs to store increases with more groups. About $20 \%$ of the nodes reach their buffer limitation in scenarios with more than four groups. With the RWP model, the average delay only increases by $16.9 \%$ as the number of groups increases from one to six. However, with the UMassBusNet model, the average delay increases by $36 \%$ as the number of groups increases from one to six groups. The larger increase is expected since the nodes in the UmassBusNet model move according to certain clusters (nodes that take the same route belong to one cluster) and hence there are more network partitions. The delivery latency for packets that need to be delivered between nodes in different clusters will be larger.

Third, we observe as before that the delivery performance using UMassBusNet model is poorer than that with the RWP model. This poorer performance is due to two factors:

- the mean intercontact time for the UMassBusNet trace is longer

- $\quad$ nodes in the UMassBusNet model move according to certain routes and hence there are more network partitions in the UMassBusNet model than in the RWP model.

As discussed before, the data efficiency with the UMassBusNet model is higher than that with the RWP model because only those packets that require fewer hops are successfully delivered in the UMassBusNet model. The average number of hops is 2.1 for the UMassBusNet model but 3 for the RWP model.

\subsection{Impact of different percentage of DTN nodes}

In some scenarios, not all nodes may support DTN functionalities. Nodes that do not support DTN functionalities can not be message ferries. Thus, in this section, we investigate the performance difference when not all nodes support DTN functionalities. We use one multicast group with 12 receivers where the multicast source generates packets at a rate of $1 \mathrm{pkt} / \mathrm{s}$. We compare the case where $50 \%$ of the nodes are DTN nodes with the case where all nodes are DTN nodes. Table 4 tabulates our results for different network scenarios. From Table 4, we see that with only $50 \%$ of the nodes being the DTN nodes, the delivery ratio drops by $10 \%$ in the $2000 \times 2000 \mathrm{~m}^{2}$ scenario but it drops by $14-16 \%$ when the network becomes very sparse (for the $3000 \times 3000 \mathrm{~m}^{2}$ and $4000 \times 4000 \mathrm{~m}^{2}$ scenarios). With fewer nodes that support DTN functionalities, there are fewer chances of using message ferrying to deliver packets across partitioned networks. Therefore, the delivery ratio is poorer with $50 \%$ of the nodes being the DTN nodes. The average delay drops with fewer nodes supporting DTN functionality. Such a drop is misleading because packets that are more difficult to be delivered are being dropped in the $50 \%$ DTN nodes scenario but are delivered (with much higher delay) when all nodes support DTN functionality. The data efficiency is only slightly worse with $50 \%$ nodes supporting DTN functionality. This is due to the increasing number of retransmissions when only $50 \%$ of the nodes support DTN functionality.

Table 4 Impact of having different percentage of nodes supporting DTN functionalities

\begin{tabular}{|c|c|c|c|c|c|c|}
\hline \multirow{2}{*}{$\begin{array}{l}\text { Simulation } \\
\text { area }\left(\mathrm{m}^{2}\right)\end{array}$} & \multicolumn{2}{|c|}{ Delivery ratio } & \multicolumn{2}{|c|}{ Average delay (s) } & \multicolumn{2}{|c|}{$\begin{array}{c}\text { Data efficiency and } \\
\text { overall efficiency }\end{array}$} \\
\hline & $50 \%$ & $100 \%$ & $50 \%$ & $100 \%$ & $50 \%$ & $100 \%$ \\
\hline $1000 \times 1000$ & 0.93 & 0.93 & 81 & 81 & $\begin{array}{c}0.40 / \\
0.18\end{array}$ & $\begin{array}{l}0.40 / \\
0.18\end{array}$ \\
\hline $2000 \times 2000$ & 0.81 & 0.90 & 2256 & 2327 & $\begin{array}{l}0.34 / \\
0.14\end{array}$ & $\begin{array}{l}0.36 / \\
0.14\end{array}$ \\
\hline $3000 \times 3000$ & 0.73 & 0.85 & 2567 & 2700 & $\begin{array}{l}0.31 / \\
0.11\end{array}$ & $\begin{array}{l}0.31 / \\
0.11\end{array}$ \\
\hline $4000 \times 4000$ & 0.67 & 0.80 & 3613 & 3725 & $\begin{array}{l}0.24 / \\
0.10\end{array}$ & $\begin{array}{c}0.27 / \\
0.10\end{array}$ \\
\hline
\end{tabular}

\subsection{Impact of different maximum node speeds}

In this section, we explore how the different $\left(v_{\min }, v_{\max }\right)$ values affect the data delivery performance. We use two network scenarios: 40 nodes distributed over

- $4000 \times 4000 \mathrm{~m}^{2}$

- $3000 \times 3000 \mathrm{~m}^{2}$.

One multicast group with 12 receivers is used. The multicast source generates packets at a rate of $1 \mathrm{pkt} / \mathrm{s}$. The multicast receivers are randomly chosen among the 40 nodes. Figure 8(a)-(c) plot the delivery ratio, average delay and data efficiency for the different $\left(v_{\min }, v_{\max }\right)$ values. From the results, we can see that the delivery ratio increases slightly initially with increasing node speeds but the average delay decreases significantly with increasing node speed. As a node moves around faster, it encounters more nodes and hence is able to disseminate messages better. As a result, the average delay decreases due to shorter mean intercontact time. However, the contact duration will become shorter too with increasing node speed. As long as the contact duration is long enough to allow nodes to transfer queued packets when they meet one another, the delivery ratio will not degrade but may improve (since the same forwarding node can be used for multiple packets). 
Figure 8 (a) Delivery ratio vs. node speed; (b) average delay vs. node speed and (c) data efficiency vs. node speed

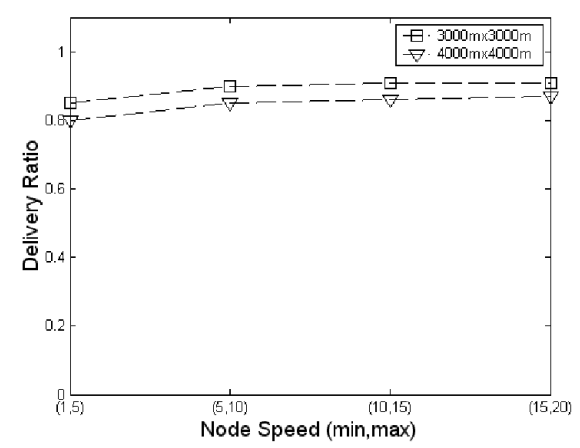

(a)

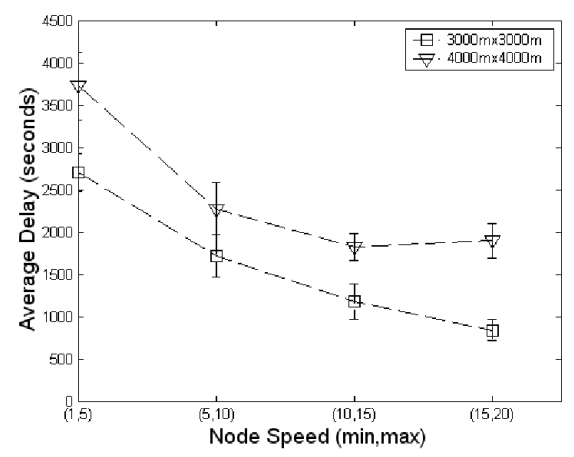

(b)

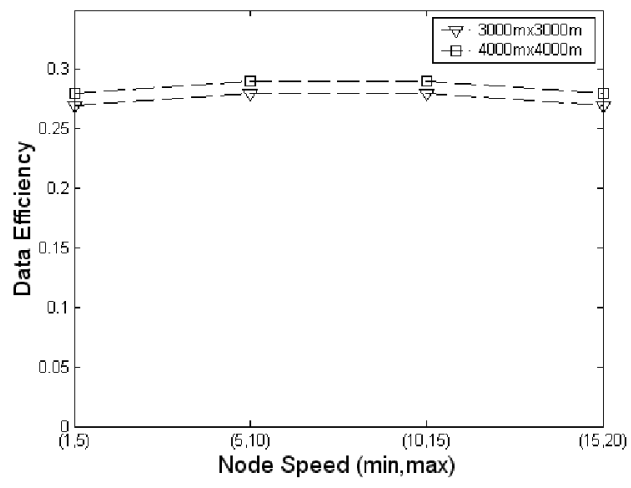

(c)

\section{Conclusion}

In this paper, we have developed a context-aware adaptive multicast routing scheme for DTNs. Our scheme is flexible and can adapt to different network environments e.g., different node densities, different mobility models. We have evaluated our CAMR scheme and compare it with other existing proposed multicast routing schemes for DTNs. Our results show that the CAMR scheme is more flexible and can provide the highest delivery ratio with lower average delay (comparing only those common packets that are delivered in all schemes) especially when the network becomes sparser. Via simulation studies, we also have shown that CAMR scales well with increasing number of groups and maintains a high delivery ratio even with increasing node speed. We also have shown that the UMassBusNet mobility model results in the poorest delivery performance due to its large intercontact time. We hope to implement the CAMR scheme in a DTN testbed for empirical evaluations.

\section{Acknowledgement}

This work is sponsored by Defense Advanced Research Projects Agency (DARPA) under contract W15P7T-05-CP413. Any opinions, findings, and conclusions or recommendations expressed in this material are those of the authors and do not necessarily reflect the views of DARPA. This document is approved for public release, unlimited distribution.

\section{References}

Bae, S., Lee, S.J., Su, W. and Gerla, M. (2000) 'The design, implementation, and performance evaluation of the on-demand multicast routing protocol in multihop wireless networks', IEEE Network, January, pp.70-77.

Broch, J. et al. (1998) 'A performance comparison of multihop wireless adhoc network routing protocol', Proceedings of ACM/IEEE Mobicom, October, pp.85-97.

Burgess, J., Gallagher, B., Jensen, D. and Levine, B.N. (2006) 'MaxProp: routing for vehicle-based disruption-tolerant networking', Proceedings of IEEE Infocom, April, pp.1-11.

Burleigh, S., Hooke, A., Torgeson, L., Fall, K., Cerf, V., Durst, B., Scott, K. and Weiss, H. (2003) 'Delay tolerant networking - an approach to interplanetary internet', IEEE Communications Magazine, June.

Cerf, V., Burleigh, S., Hooke, A., Torgeson, L., Durst, R., Scott, K., Fall, K. and Weiss, H. (2007) Delay-Tolerant Network Architecture, IRTF RFC4838, April.

Cerpa, A., Elson, J., Estrin, D., Girod, L., Hamilton, M. and Zhao, J. (2001) 'Habitat monitoring: application driver for wirelss communications technology', Proceedings of ACM Sigcomm Workshop on Data Communications, April, Costa Rica.

Chuah, M., Cheng, L. and Davison, B.D. (2005) 'Enhanced Disruption and Fault Tolerant Network Architecture for Bundle Delivery (EDIFY)', Proceedings of IEEE Globecom, November, St Louis, pp.807-812.

Dai, F. and Wu, J. (2007) 'Logarithmic store-carry-forward routing in mobile ad hoc networks', IEEE Transactions on Parallel and Distributed Systems, Vol. 18, No. 6, June, pp.735-748.

DARPA (2005) Disruption Tolerant Networks Program http://www.darpa.mil/ato/solicit/dtn/, accessed on 3rd August.

Fall, K. (2003) 'A delay-tolerant network architecture for challenged internets', Proceedings of ACM SIGCOMM, Germany, August, pp.27-34.

Fall, K. (2004) Messaging in Difficult Environments, Intel Research Berkeley, IRB-TR-04-019, Decemeber.

Fall, K., Hong, W. and Madden, S. (2003) Custody Transfer for Reliable Delivery in Delay Tolerant Networks, IRB-TR-03-030, July, http://dtnrg.org/wiki/docs/papers/ custody-xfer-tr.pdf

Jain, S., Demmer, M., Patra, R. and Fall, K. (2005) 'Using redundancy to cope with failures in a delay tolerant network', ACM SIGCOMM Computer Communication Review, October, pp.109-120. 
Jain, S., Fall, K. and Patra, R. (2004) 'Routing in a delay tolerant networks', Proceedings of ACM SIGCOMM, New York, pp.145-158.

Lindgren, A., Doria, A. and Schelen, O. (2003) 'Probabilistic routing in intermittently connected networks', Sigmobile, Mobile Computing and Communications Review, Vol. 7, No. 3, pp.19-20.

Malladi, R. and Agrawal, D.P. (2002) 'Curent and future applications of mobile and wireless networks', Communications of the ACM, Vol. 45, pp.144-146.

Moy, J. (1994) Multicast Extension to OSPF, IETF RFC 1584.

Navidi, W. (2006) Statistics for Engineers and Scientists, McGraw-Hill, ISBN-10 0073309494.

Spyropoulos, T., Psounis, K. and Raghavendra, C.S. (2005) 'Spray and wait: an efficient routing scheme for intermittently connected mobile networks', Proceedings of ACM SIGCOMM Workshop on Delay Tolerant Networking, Philadelphia, PA, pp.252-259.

Waitzman, D., Patridge, C. and Deering, S. (1988) Distance Vector Multicast Routing Protocol (DVMRP), IETF RFC 1075.

Wang, Y., Jain, S., Martonosi, M. and Fall, K. (2005) 'Erasure-coding based routing for opportunistic networks', Proceedings of ACM SIGCOMM Workshop on Delay-Tolerant Networking, August, pp.229-236.

Wu, H., Fujimoto, R. and Riley, G. (2004) 'Analytical models for data dissemination in vehicle to vehicle networks', Proceedings of IEEE, VTC Fall.

Xie, J., Talpade, R.R., Mcauley, A. and Liu, M.Y. (2002) 'AMRoute: ad hoc multicast routing protocol', Mobile Networks and Applications, Vol. 7, No. 6, pp.429-439.
Ye, Q., Cheng, L., Chuah, M.C. and Davison, B. (2006) 'OS-Multicast: on-demand situation-aware multicasting in disruption tolerant networks', Proceedings of IEEE 63rd Vehicular Technology Conference, May, Melbourne, Australia, pp.96-100.

Zhang, X., Kurose, J., Levine, B.N., Towsley, D. and Zhang, H. (2007) 'Study of a bus-based disruption tolerant network: mobility modeling and impact on routing', Proceedings of 13th ACM Mobicom, Montreal, Canada, pp.195-206.

Zhang, Z. and Zhang, Q. (2007) 'Delay/disruption tolerant mobile ad hoc networks: latest developments', Wireless Communications and Mobile Computing, Vol. 7, No. 10, December, pp.1219-1232.

Zhao, W., Ammar, M. and Zegura, E. (2004) 'A message ferrying approach for data delivery in sparse mobile ad hoc networks', Proceedings of ACM Mobicom, May, Tokyo, Japan, pp.187-198.

Zhao, W., Ammar, M. and Zegura, E. (2005) 'Multicasting in delay tolerant networks: semantic models and routing algorithms', Proceedings of ACM SIGCOMM Workshop on Delay-Tolerant Networking, Philadelphia, PA, pp.268-275.

\section{Website}

UCB/LBNL/INT 'The Network Simulator $n s-2$ ', Online at http://www.isi.edu/nsnam/ns/ 